\title{
QUOTIENT RINGS OF A RING AND A SUBRING WHICH HAVE A COMMON RIGHT IDEAL
}

\author{
JAY SHAPIRO
}

\begin{abstract}
Let $R$ be a subring of $S$ and let $A \subseteq R$ be a right ideal of $S$. In this paper we show that there is a bijection between right torsion theories $\tau$ over $S$ such that $A$ is $\tau$-dense and right torsion theories $\sigma$ over $R$ such that $S / A$ is $\sigma$-torsion. $A$ similar result is obtained for the left side with the bijection between torsion theories over $S$ with $S A$ dense and torsion theories over $R$ with $R A$ dense. It is also shown that the ring of quotients of $R$ and $S$ at these corresponding torsion theories are equal. As a corollary, when $A$ is chosen appropriately $R$ and $S$ have the same right (left) maximal quotient ring.
\end{abstract}

Introduction. In this paper, all rings have units and all modules and subrings are unital.

Let $S$ be a ring and let $A$ be a right ideal of $S$. The idealizer of $A$ in $S$, denoted $I(A)$, is the largest subring of $S$ containing $A$ as a two-sided ideal. Armendariz and Fisher [1] have shown that with various assumptions on $A, S$ being semiprime right (left) Goldie is equivalent to $I(A)$ being semiprime right (left) Goldie. They have also shown that under certain circumstances, the two rings have the same classical ring of quotients. In a similar vein Goodearl [3] has shown that if $S$ is right nonsingular and if $A$ is essential in $S$, then $I(A)$ is right nonsingular and has the same right maximal quotient ring as $S$.

Teply [5] has extended these results and shown that one need only assume that the subring be a subidealizer of $A$ (a subidealizer of $A$ is a subring of $I(A)$ which contains $A$ ). The purpose of this paper is to generalize and add to these results in various directions. First, we assume only that the subring, denoted as $R$, contains $A$ as a right ideal. Also, we work with arbitrary torsion theories. tors- $R$ (resp. $R$-tors) will denote the collection of left (resp. right) torsion theories over the ring $R$. In the first section we show that there is a bijection between the set $\{\sigma \in \operatorname{tors}-R \mid S / A$ is $\sigma$-torsion $\}$ and the set $\{\tau \in$ tors- $S \mid S / A$ is $\tau$-torsion $\}$. It is then shown that the ring of quotients of $R$ and $S$ at their corresponding torsion theories are equal. We also investigate which properties of a torsion theory transfer under this bijection. In $\$ 2$ similar results are obtained for left torsion theories over $S$ and $R$. Finally, it is shown that if $A$ is chosen appropriately, $S$ and $R$ have the same right (left) maximal quotient ring.

Let $X$ be a subset of a ring $T$ and let $t \in T$. Then $X t^{-1}=\{r \in T \mid r t \in X\}$. Similarly one defines $t^{-1} X$. Since we will be dealing with two rings, one of which is contained in the other, there is the possibility of confusion with this notation.

Received by the editors September 12, 1979 and, in revised form, January 8, 1980.

1980 Mathematics Subject Classification. Primary 16A08, 16A63. 
However, $X$ will always be a one-sided ideal of at least one of the rings. Therefore, the $T$ in the above definition of $X t^{-1}$ will be the largest ring containing $X$ as a one-sided ideal. We assume the reader is familiar with the notion of a torsion theory and its module of quotients (see [2], [4]). Recall that a collection of right ideals $\mathcal{T}$ of a ring $S$ is called an idempotent filter of right ideals if it satisfies the following:

(T1) $A \in \mathcal{T} \Rightarrow t^{-1} A \in \mathcal{T}$ for all $t \in S$.

(T2) If $B \subset A$ are right ideals in $S$ such that $A \in \mathcal{T}$ and $a^{-1} B \in \mathcal{T}$ for each $a \in A$, then $B \in \mathcal{T}$.

(T3) If $A \in \mathcal{T}$ and $A \subseteq B$ then $B \in \mathcal{T}$.

Given a torsion theory $\tau$ over $S$ a right ideal $A$ is called $\tau$-dense if $S / A$ is $\tau$-torsion. There is a bijection between right torsion theories over $S$ and idempotent filters of right ideals of $S$ given by $\tau \rightarrow \mathcal{T}$, where $\mathcal{T}$ is the set of $\tau$-dense right ideals. Given a right module $M, \tau(M)$ will denote the unique maximal $\tau$-torsion submodule of $M . M_{\tau}$ will denote the module of quotients at $\tau$. Since there is a bijection between idempotent filters and torsion theories we will also talk about the module of quotients of $M$ at an idempotent filter.

An injective module $E$ is said to cogenerate a torsion theory $\tau$ if the class of $\tau$-torsion free modules is equal to the class of modules which embed in a power of $E$. Every injective module cogenerates a torsion theory and every torsion theory is cogenerated by some injective module.

\section{Right torsion theories.}

Definition. Let $A$ be a right ideal of $S$. Then a subring $R$ of $S$ is called an $A$-subring of $S$ if $R$ contains $A$ as a right ideal.

Throughout this paper $S$ will be a ring, $A$ a right ideal of $S$ and $R$ an $A$-subring of $S$. We will assume that $A$ is nonzero for otherwise the results are trivial.

TheORem 1.1. Given $S, A$ and $R$ let $\tau \in$ tors-S with idempotent filter $\mathcal{T}$ such that $A \in \mathcal{T}$. Let $\mathcal{E}=\left\{B_{R} \subseteq R \mid H \cap A \subseteq B\right.$ for some $\left.H \in \mathcal{T}\right\}$. Then $\mathcal{L}$ is an idempotent filter of right ideals and $S_{\tau}$ is the ring of quotients of $R$ with respect to $\mathcal{L}$.

Proof. First observe that for a right ideal $H$ of $S$ and any $r \in R, r^{-1}(H \cap R)=$ $\left(r^{-1} H\right) \cap R$. Hence, $\mathcal{E}$ satisfies (T1). Let $B \in \mathcal{L}$, i.e., there exist $H \in \mathcal{T}$ such that $H \cap R \subseteq B$. Then $H \cap A$ is a right ideal of $S$, is $\tau$-dense, and is contained in $B$. Therefore, $B S$ is an element of $\mathcal{T}$ and $B A$ is an element of $\mathcal{L}$. We also see that every element of $\mathcal{L}$ contains an element of $\mathcal{T}$. Now assume that $D \subseteq C$ are right ideals of $R$ such that $C \in \mathcal{L}$ and every element of $C / D$ is annihilated by some element of $\mathcal{L}$. Then every element of $C / D A$ is annihilated by some element of $\mathcal{L}$. Viewing $C / D A$ as a subset of the $S$ module $S / D A(D A$ after all is a right ideal of $S$ ) we see that every element of $C / D A$ is $\tau$-torsion. Since $\mathcal{T}$ is an idempotent filter and $C S \in \mathcal{T}$ we have $D A$ is an element of $\mathcal{T}$. Hence, $D \in \mathcal{L}$ showing $\mathcal{E}$ satisfies (T2). Clearly (T3) is satisfied so $\mathcal{E}$ is a filter.

By the above it is clear that for any right $S$ module $M, \sigma(M)=\tau(M)$. In particular, there is a ring homomorphism $R \rightarrow S_{\tau}$ whose kernel is equal to $\sigma(R)$. By identifying $R$ and $S$ with their images in $S_{\tau}$ we can assume that they are both 
torsion free. Let $t \in S$. Clearly $t\left(t^{-1} A \cap R\right) \subseteq A \subset R$. Since $t^{-1} A \cap R \in \mathcal{E}$ it follows that $t\left(t^{-1} A \cap R\right) \neq 0$. Therefore, $S$ is an essential extension of $R$ and $S / R$ is $\sigma$-torsion. Since $S_{\tau} / S$ is $\sigma$-torsion, $S_{\tau} / R$ is $\sigma$-torsion. If $E$ is an essential extension of $S$ as an $S$ module it is $\sigma$-torsion free. So by an argument similar to the one above $E$ is an essential extension as an $R$ module. Thus, if $E(S)$ is the injective hull of $S$ as a right $S$ module, it is also the injective hull of $S$ as a right $R$ module. Therefore, to show that the map $R \rightarrow S_{\tau}$ is the ring of quotients of $R$ with respect to $\sigma$ it suffices to prove that $E(S) / S_{\tau}$ is $\sigma$-torsion free. However, since we already know that it is $\tau$-torsion free, we are done.

Proposition 1.2. With $S, A$ and $R$ as usual let $\sigma \in$ tors- $R$ with idempotent filter $\mathcal{L}$ such that $S / A$ is $\sigma$-torsion. Then the collection of right ideals $\mathcal{T}$ of $S$, defined by $H \in \mathcal{T}$ if and only if $H \cap R \in \mathcal{E}$, is an idempotent filter.

Proof. Let $H \in \mathcal{T}$. Since $A / A \cap H \simeq(A+H) / H$, we then have the following exact sequence:

$$
0 \rightarrow(A+H) / H \rightarrow S / H \rightarrow S /(A+H) \rightarrow 0 .
$$

Since the endpoints are $\sigma$-torsion we see that $S / H$ is $\sigma$-torsion. Thus for any $t \in S, t^{-1} H$ contains an element of $\mathcal{L}$, i.e., $t^{-1} H \in \mathcal{T}$. Now let $H$ and $K$ be right ideals of $S$ such that $H \subset K, K \in \mathcal{T}$ and every element of $K / H$ is annihilated by some element of $\mathcal{T}$. Then we have the $S / K$ and $K / H$ are $\sigma$-torsion. Thus $S / H$ is $\sigma$-torsion. In particular, $1+H$ is annihilated by some $B \in \mathcal{L}$. Therefore, $H \in \mathcal{T}$. As before, (T3) is automatically satisfied.

The assignments obtained in Theorem 1.1 and Proposition 1.2 will be denoted $\tau \rightarrow \tau \cap R$ and $\sigma \rightarrow S \sigma$ respectively. It is not difficult to see that these mappings are inverses of each other. Thus, there is a bijection between the sets $\{\tau \in$ tors$S \mid S / A$ is $\tau$-torsion $\}$ and $\{\sigma \in$ tors- $R \mid S / A$ is $\sigma$-torsion $\}$.

A question that arises naturally is which properties of torsion theories (e.g. perfect) are invariant under this bijection? The next result shows that some of the more interesting properties do transfer. For the basic definitions and results on these properties see [2].

Proposition 1.3. Let $S, A, R$ and $\tau$ be as usual with $\sigma=\tau \cap R$. Then we have the following:

(i) $\tau$ is perfect $\Leftrightarrow \sigma$ is perfect;

(ii) $\tau$ is a torsion theory derived from a right Ore set $\Leftrightarrow \sigma$ is derived from a right Ore set;

(iii) the $\tau$-torsion modules form a TTF class $\Leftrightarrow$ the $\sigma$-torsion modules form a TTF class;

(iv) $\tau$ prime $\Leftrightarrow \sigma$ prime;

(v) $\tau$ semiprime $\Leftrightarrow \sigma$ semiprime;

(vi) $\tau$ exact $\Leftrightarrow \sigma$ exact

(vii) $\tau$ noetherian $\Leftrightarrow \sigma$ noetherian.

Proof. Let $\mathcal{T}$ and $\mathcal{L}$ denote the idempotent filter of right ideals associated with $\tau$ and $\sigma$ respectively. 
(i) Recall that given an arbitrary ring $T$ and torsion theory $\gamma$ on $T$ then $\gamma$ is perfect if and only if given any $\gamma$-dense right ideal $I$ of $T, I T_{\gamma}=T_{\gamma}$. With this in mind (i) then follows from the fact that every element of $\mathcal{L}$ contains an element of $\mathcal{T}$ and vice versa.

(ii) Let $X$ be the right Ore set of $S$ with torsion theory $\tau$ and let $\bar{X}=X \cap R$. Let $B \in \mathcal{L}$. Then, since $B$ contains an element of $\mathcal{T}, B \cap \bar{X} \neq \varnothing$. Clearly the image of every element of $\bar{X}$ is invertible in $S_{\tau}$. Now let $q$ be an arbitrary element of $S_{\tau}$. Identifying $R$ with its image in $S_{\tau}$, there exists $B \in \mathcal{L}$ such that $q B \subseteq R$. Therefore, there is some $t \in \bar{X}$ such that $q t=r \in R$, i.e. $q=r t^{-1}$. Thus, $S_{\tau}$ is the ring of quotients of $R$ with respect to $\bar{X}$.

In the other direction, assume $Y$ is a right Ore set of $R$ with torsion theory $\sigma$. By Theorem 1.1 it is clear that the image of every element of $Y$ is invertible in $S_{\tau}$, and any $q \in S_{\tau}$ can be written in the form $a y^{-1}$ where $a$ is in the image of $S$ and $y$ is in the image of $Y$.

(iii) It is known that the o-torsion modules form a TTF class if and only if $\mathcal{L}$ contains a unique minimal element [4, Proposition VI, 6.12]. Thus, if $B$ is the unique minimal element of $\mathcal{E}$, since $B$ contains an element of $\mathcal{T}, B$ must also be the unique minimal element of $\mathcal{T}$. For the converse, observe that if $B$ is the unique minimal element of $\mathcal{T}$, then $B \subseteq A$.

(iv) If $M$ is a $\tau$-torsion free $S$-module, then it follows from the proof of Theorem 1.1 that $M$ is injective as an $S$-module ( $\tau$-injective) if and only if $M$ is injective as an $R$-module ( $\sigma$-injective). Now let $M$ be a $\tau$-cocritical $S$-module (o-cocritical $R$-module) whose injective hull cogenerates $\tau(\sigma)$. Then $M(M A)$ is a $\sigma$-cocritical $R$-module ( $\tau$-cocritical $S$-module) such that $E_{S}(M)=E_{R}(M)\left(E_{R}(M)=E_{S}(M A)\right.$ ).

(v) follows from (iv) and the fact that $\tau \rightarrow \tau \cap R$ is order preserving.

(vi) Assume $\sigma$ is exact. Let $E$ be a $\tau$-torsion free, $\tau$-injective $S$-module with $E^{\prime}$ an $S$-submodule of $E$ such that $E / E^{\prime}$ is $\tau$-torsion free. Then $E / E^{\prime}$ is $\sigma$-torsion free, and therefore, $\sigma$-injective $[2,16.1]$. Hence, $E / E^{\prime}$ is $\tau$-injective proving that $\tau$ is exact $[2,16.1]$. The converse is immediate since $M_{\tau}=M_{\sigma}$ for all $S$-modules $M$.

(vii) This follows from the fact that every element of $\mathcal{L}$ contains an element of $\mathcal{T}$ and conversely.

A ring $R$ is said to be a right Ore ring if the set of regular elements of $R$ is a right Ore set, i.e., $R$ has a right classical quotient ring. The next result follows immediately from Theorem 1.1 and Proposition 1.3(ii).

Corollary 1.4. Let $S$ be $a$ ring and let $A$ be a right ideal of $S$ such that $A$ contains a regular element. Let $R$ be any $A$-subring of $S$. Then $S$ is a right Ore ring only if $R$ is a right Ore ring.

2. Left torsion theories. We now want to obtain analogous results for left torsion theories over $S$ and $R$. In the following $S, A$ and $R$ will remain the same but all torsion theories will be on the left. The results on this side are very similar to the results, already obtained for the right side. In some instances the proofs are also similar, in which case we refer the reader to the appropriate place. 
THEOREM 2.1. Let $\tau \in S$-tors such that $S A$ is $\tau$-dense. Then the collection, $\mathcal{E}=\left\{{ }_{R} B \subseteq R \mid S B\right.$ is $\tau$-dense $\}$, is an idempotent filter and $S_{\tau}$ is the ring of quotients of $R$ at $\mathcal{L}$.

Proof. We first want to show that if $B \in \mathcal{L}$ and $r \in R$ then $B r^{-1} \in \mathcal{L}$, i.e., $S\left(B r^{-1}\right)$ is $\tau$-dense. Since $(S B) r^{-1}$ is $\tau$-dense, it suffices to show that $S A\left[(S B) r^{-1}\right] \subseteq$ $S\left(B r^{-1}\right)$. Let $x \in(S B) r^{-1}$; thus $x r=\sum_{i=1}^{n} s_{i} b_{i}$ where $s_{i} \in S, b_{i} \in B$. Let $a \in A$; then $a x r=a \sum_{i=1}^{n} s_{i} b_{i} \in B$, since $a s_{i} \in A \subseteq R$. Therefore, we have that $a x \in$ $B r^{-1}$. So for any $s \in S$, sax $\in S\left(B r^{-1}\right)$.

Now let $B \in \mathcal{L}$, and let $D$ be a left ideal of $R$ which is contained in $B$ such that every element of $B / D$ is annihilated by some element of $\mathcal{L}$. Let $t \in S, b \in B$ and $C=D b^{-1}$. Then $(S C) t^{-1}$ is $\tau$-dense and $(S C) t^{-1} t b \subseteq S C b \subseteq S D$. Thus, the $S$ module $S B / S D$ is $\tau$-torsion. Hence, $S D$ is $\tau$-dense, proving that $\mathcal{L}$ satisfies (T2), with (T3) again immediate.

Let $\sigma$ denote the torsion theory associated to $\mathcal{L}$. As before we have that, for any left $S$ module $M, \sigma(M)=\tau(M)$. So $S_{\tau}$ is $\sigma$-torsion free and there is a ring map $R \rightarrow S_{\tau}$ with kernel $\sigma(R)$. Again we identify $R$ and $S$ with their images in $S_{\tau}$. Let $q \in S_{\tau}$. Then there exists $H$, a $\tau$-dense left ideal of $S$, such that $H q \subseteq S$. Observe that $R A H \subset R$ and is $\sigma$-dense. Since $0 \neq(R A H) q \subseteq R A(H q) \subseteq(R A S)=R a$ we have that $S_{\tau}$ is an essential extension of $R$ and $S_{\tau} / R$ is $\sigma$-torsion. The remainder of the proof proceeds as in Theorem 1.1.

Proposition 2.2. With $S, A$ and $R$ as usual let $\sigma \in R$-tors $R$ with idempotent filter $\mathcal{L}$ such that $R A \in \mathcal{L}$. Then $\mathcal{T}=\left\{{ }_{s} H \subseteq S \mid H \cap R \in \mathcal{L}\right\}$ is an idempotent filter.

Proof. First note that the left $R$ module $S / R A$ is $\sigma$-torsion since it is annihilated by $R A$. Let $H$ be any left ideal of $S$ that is in $\mathcal{T}$. Since $R A / R A \cap H$ is $\sigma$-torsion and $R A \cap H \subseteq H$, we have that $S / H$ is also $\sigma$-torsion. Now the proof proceeds as in Proposition 1.2.

The assignments that we obtained in Theorem 2.1 and Proposition 2.2 will be denoted $\tau \rightarrow \tau \cap R$ and $S \rightarrow S \sigma$ respectively. As in $\S 1$, it follows that there is a bijection between the sets $\{\tau \in S$-tors $\mid S A$ is $\tau$-dense $\}$ and $\{\sigma \in R$-tors $\mid R A$ is o-dense \}.

REMARK. As on the right, certain properties of torsion theories are preserved under this bijective correspondence. We note the following where $\sigma=\tau \cap R$ :

(i) $\tau$ perfect $\Leftrightarrow \sigma$ perfect. This follows as in Proposition 1.3(i).

(ii) $\tau$ bounded $\Leftrightarrow \sigma$ bounded. The proof of $\Rightarrow$ is routine. While in the other direction one just has to note that if $B$ is a $\sigma$-dense (two-sided) ideal of $R$, so is $B A \subseteq B$. Thus, $S B A$ is a $\tau$-dense (two-sided) ideal of $S$.

(iii) If $\sigma$ is derived from a left Ore set then so is $\tau$. This follows as in Proposition 1.3(ii).

(iv) $\tau$ prime $\Leftrightarrow \sigma$ prime. The proof in one direction is similar to the proof in Propositon 1.3(iv). For the other direction let $M$ be a $\sigma$-cocritical $R$-module. Then $M$ is contained in the $S$-module $M_{\sigma}$. Thus $S M$ is well defined. Let $0 \neq{ }_{s} N \subseteq S M$. Then $N \cap M \neq 0$, so $M / N \cap M$ is $\sigma$-torsion. Let $t=\Sigma s_{i} m_{i} \in S M$. For each $i$ 
there exists $B_{i} \in \mathcal{L}$ such that $B_{i} m_{i} \in N \cap M$. Thus, $\cap\left(S B_{i}\right) s_{i}^{-1} \subseteq S(N \cap M) \subseteq$ $N$. Therefore, $S M / N$ is $\tau$-torsion, proving that $S M$ is $\tau$-cocritical. Clearly $E_{R}(M)$ $=E_{S}(S M)$ so we are done.

(v) $\tau$ semiprime $\Leftrightarrow \sigma$ semiprime.

(vi) $\tau$ noetherian $\Leftrightarrow \sigma$ noetherian. Assume that $\tau$ is noetherian and let $I_{1} \subseteq I_{2}$ $\subseteq \cdots$ be an ascending chain of left ideals of $R$ such that $\cup I_{n} \in \mathcal{L}$. Since $S\left(\cup I_{n}\right) \subseteq \cup S I_{n}$ there exists an integer $m$ such that $S I_{m} \in \mathcal{T}$. Thus $I_{m} \in \mathcal{L}$. Now let $\sigma$ be noetherian. Then the direct sum of $\tau$-injective, $\tau$-torsion free $S$ modules is $\sigma$-injective (this is the same as on the right side). Thus, $\tau$ is noetherian $[2,14.1]$.

(vii) $\sigma$ exact $\Leftrightarrow \tau$ exact. This is the same as Proposition 1.3(vii).

Note, the converse to (iii) is false as shown by the following simple example. Let $S=M_{2}(K), K$ a field, and let $A=\left(\begin{array}{ll}K & K \\ 0 & 0\end{array}\right)$. So $R=\left(\begin{array}{ll}K & K \\ 0 & K\end{array}\right)$ is an $A$-subring of $S$. Then $S$ is (trivially) a localization of itself at a left Ore set and $S=S A$ is dense with respect to this torsion theory. Yet $S$ is not a classical localization of $R$.

For any ring $T$, an important torsion theory is the one cogenerated by the injective hull of the ring when considered as a module over itself. This torsion theory is called the Lambek torsion theory and the quotient ring is called the maximal quotient ring of $T$. Clearly there are distinct notions of right and left maximal quotient ring.

Corollary 2.3. Let $S, A$ and $R$ be as usual. Then we have the following:

(i) $A$ dense in the right Lambek torsion theory of $S$ implies that $R$ and $S$ have the same right maximal quotient ring;

(ii) $S A$ dense in the left Lambek torsion theory implies $R$ and $S$ have the same left maximal quotient ring.

Proof. (i) The maximal quotient ring of any ring $R$ satisfies the following two properties: The canonical map from $R$ into its quotient ring is an embedding. If $T$ is any ring of quotients of $R$ such that the canonical map $R \rightarrow T$ is an embedding, then $R$ and $T$ have the same maximal quotient ring. Now let $R$ and $S$ be as usual with $Q$ the maximal quotient ring of $S$. By Theorem 1.1, $Q$ is a ring of quotients of $R$ such that the map $R \rightarrow Q$ is an embedding. Since $Q$ is its own right maximal quotient ring we are done.

(ii) Using Theorem 2.1 the proof is the same as in (i).

Recall that a ring is said to be right nonsingular if every essential right ideal has its left annihilator equal to zero. If two rings have isomorphic maximal quotient rings then one is nonsingular if and only if the other is nonsingular [4, Proposition XII, 2.2]. Thus, Corollary 2.3 also says that if $A$ ( $S A$ as a left ideal) is dense in the right (resp. left) Lambek torsion theory then $R$ is right (left) nonsingular if and only if $S$ is right (left) nonsingular.

It is known that a two-sided ideal is dense in the right (left) Lambek torsion theory if and only if its left (right) annihilator is equal to zero. Thus the next result is just a special case of Corollary 2.3. However, we state it separately as we feel it is of sufficient interest. 
COROLlary 2.4. Let $S$ be a semiprime left Goldie ring. Let $A$ be a right ideal of $S$ with right annihilator equal to zero, and let $R$ be an $A$-subring of $S$. Then $R$ is left nonsingular and has a semisimple maximal quotient ring.

Recall the example of the ring of $2 \times 2$ matrices over a field and its subring of upper triangular matrices. This example shows that the above result is the best possible. That is, in general we cannot hope to show that $R$ is semiprime. However, it was shown in [1, Theorem 2.1] that if we assume the left annihilator of $A$ is zero and $R$ is the idealizer of $A$, then $R$ is in fact semiprime left Goldie.

\section{REFERENCES}

1. E. P. Armendariz and J. W. Fisher, Idealizers in rings, J. Algebra 39 (1976), 551-562.

2. J. S. Golan, Localization of noncommutative rings, Marcel Dekker, New York, 1975.

3. K. R. Goodearl, Idealizers and nonsingular rings, Pacific J. Math. 48 (1973), 395-402.

4. B. Stenstrom, Rings of quotients, Springer-Verlag, New York, 1975.

5. M. L. Teply, Prime singular splitting rings with finiteness conditions, Lecture Notes in Math., vol. 545, Springer-Verlag, Berlin, 1976.

Department of Mathematics, Wayne State University, Detrort, Michigan 48202 